\section{BET INHIBITORS SYNERGIZE WITH ANTI-PD1 BY RESCUING TCF1+ PROGENITOR EXHAUSTED T CELLS IN ACUTE MYELOID LEUKEMIA}

${ }^{1}$ Kyle Romine, ${ }^{1}$ Hyun-Jun Cho, 'Y Yoko Kosaka, ${ }^{2}$ Kaelan Byrd, ${ }^{1} J e s s e$ Coy, ${ }^{1}$ Patrick Flynn, ${ }^{3}$ Matthew Newman, ${ }^{1}$ Christopher Loo, ${ }^{1}{ }^{4}$ Evan Lind*. ${ }^{1}$ Oregon Health \& Science University, Porltand, OR, United States; ${ }^{2}$ Oregon Health and Sciecnes University, Portand, OR, United States; ${ }^{3}$ Oregon Health \& Science Unversity, Portland, OR, United States; ${ }^{4} \mathrm{OHSU}$, Portland, United States

Background Acute Myeloid Leukemia (AML) is the most common adult leukemia and has a very poor prognosis. With a 5year survival of under $30 \%$ (seer.cancer.gov), most people diagnosed with AML will die from the disease. AML is caused by an uncontrolled proliferation of poorly differentiated myeloid precursor cells which results from a combination of three classes of mutations that affect proliferation, differentiation and epigenetic state. For this reason, drugs targeting epigenetic modifications are being actively studied in AML. AML has been shown to avoid immune recognition though inhibiting the function of multiple cell types, especially $\mathrm{T}$ cells $^{1}{ }^{2}$ and therefore immune checkpoint blockade presents a promising therapy for any immune-targeted strategy; however, clinical trials to date have shown very modest efficacy. ${ }^{3-5} \mathrm{~T}$ cell exhaustion in cancer has been shown to be a regulated process involving transcriptional and epigenetic changes. ${ }^{6-9}$ BRD4 has been shown to be important for maintaining this exhaustion state. ${ }^{10}{ }^{11}$ It stands to reason that drugs designed to target epigenetic pathways in tumors will have effects on T cell populations present in the tumor microenvironment. In these studies, we investigated the effects of the BET inhibitor (BETi) JQ1 on T cell exhaustion and checkpoint responsiveness in a murine model of AML.

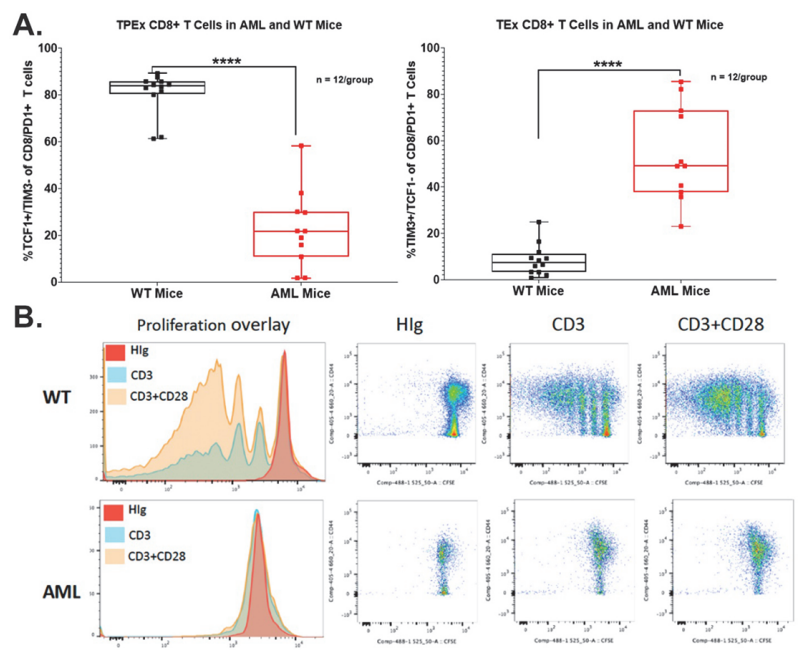

Abstract 734 Figure $1 \mathrm{~T}$ cell exhaustion in the AML mouse model. (A) Cytotoxic T cells show an exhausted phenotype in mice with AML. Spleen cultures from mice with AML or WT controls were stained with antibodies to CD3, CD8, TIM3, PD1, and TCF1. Left shows percent of TPEX CD8 T cells. Right panel shows TEX CD8 T cells. $\mathrm{N}=12$ animals per group. (B) Proliferative defect in T cells in mice with AML. Splenocytes were labeled with the proliferation dye CFSE. Whole spleen suspensions were stimulated with anti-CD3 or anti-CD3 and anti-CD28 for 3 days. FACs plots show proliferation of T cells in each condition
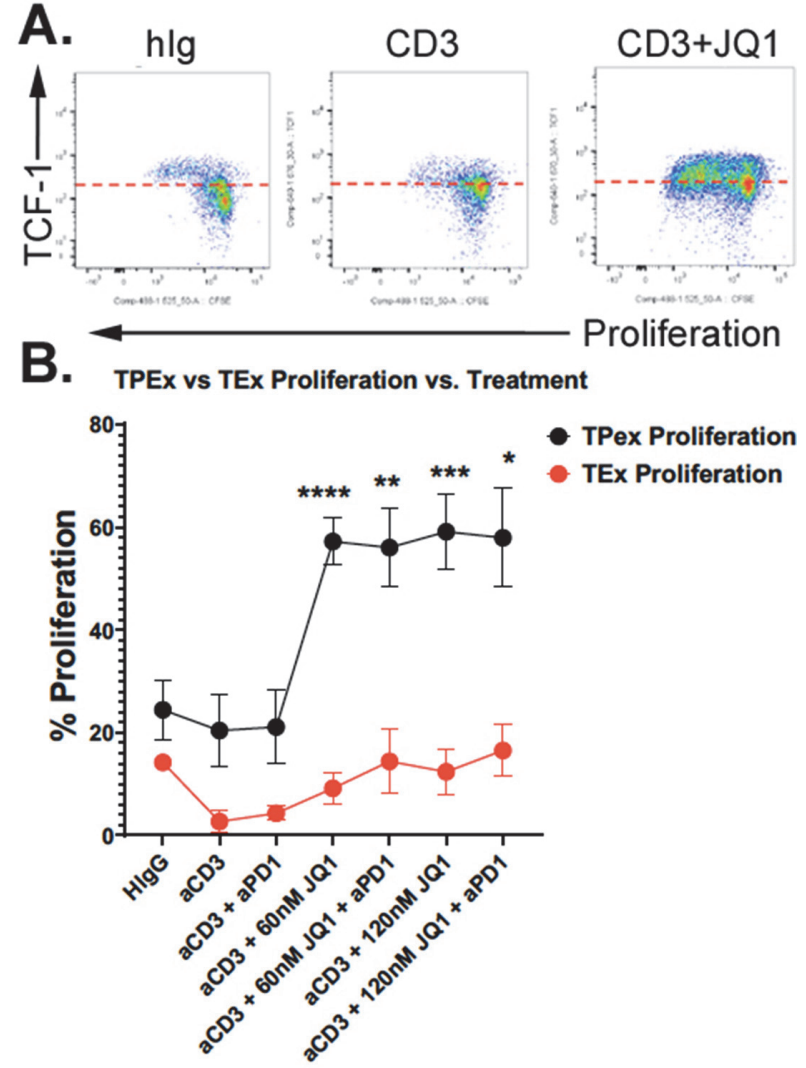

Abstract 734 Figure 2 Treatment with JQ1 results in expansion of T cells with TPEX. (A) Example of proliferation (CFSE dilution) vs TCF-1 expression showing unstimulated, CD3 or CD3+JQ1 $120 \mathrm{nM}$ in in vitro 3-day culture. Results gated on CD8 T cells. (B) Summary of T cell proliferation from 4 independent experiments showing the percent proliferation of CD8 T cells with TPEX (PD1+ Tim-3- TCF-1+) (black line) or TEX (PD1+Tim-3+TCF1-) phenotype (red line). Statistics are unpaired $\mathrm{T}$-Test for each treatment condition.

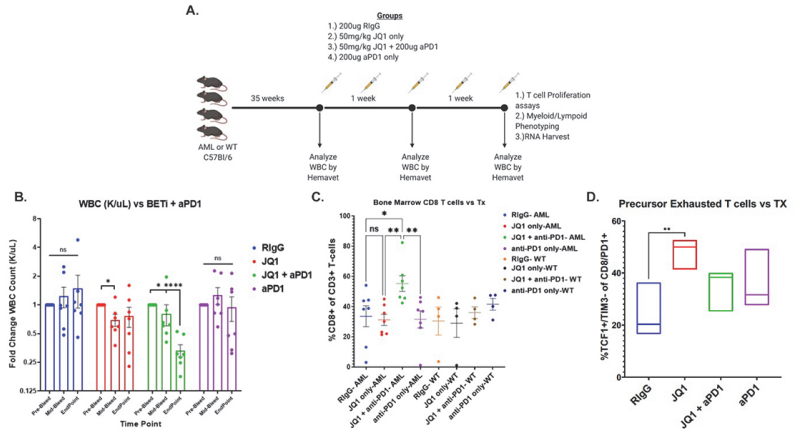

Abstract 734 Figure 3 In vivo treatment of FTL mice with the BETi JQ1. (A) Schematic overview of treatment protocol. (B) White blood cell counts at pre-treatment, 1 week and 2 weeks after JQ1, PD1 blockade or both. (C) Percent of CD8+ T cells of all CD3-gated T cells in the BM of treated animals. (D) A-C Percent of precursor-exhausted CD8+ T cells as a percent of all T cells in the spleen of treated animals. Results combined from 2 separate experiments $n=7$. $D$ One experiment $n=3$. 
Methods The AML mouse model bears FLT3-ITD and deletion of TET2 restricted to the myeloid lineage. For in vitro studies, splenocytes were stimulated with anti-CD3 and either JQ1, anti-PD1 or both and proliferation and differentiation status were assessed by flow cytometry. For in vivo studies, treatment consisted of 2 weeks with JQ1, anti-PD1 or both.

Results This mouse model of AML exhibits an expansion of terminally exhausted $\mathrm{T}$ cells and impaired proliferative capacity after stimulation through the TCR (figure 1). Ex vivo treatment with BETi and anti-PD1 reverses CD8 + T cell exhaustion via rescue of proliferative dysfunction and expansion of more functional precursor exhausted $\mathrm{T}$ cells (TPEx-CD8, PD1 + , TCF1+, TIM3-) (figure 2). Finally, we show that BETi synergizes with anti-PD1 in vivo leading to a reduction of tumor cells in multiple organ sites, and enrichment of CD8+ $\mathrm{T}$ cells in the bone marrow (figure 3).

Conclusions Using an AML mouse model that exhibits leukemia-induced immune exhaustion, we demonstrate the pre-clinical efficacy of combining BETi and anti-PD1 therapy in the treatment of AML.

\section{REFERENCES}

1. Lamble AJ, Lind EF. Targeting the immune microenvironment in acute myeloid leukemia: a focus on T Cell immunity. Front Oncol 2018;8:213.

2. Lamble AJ, Kosaka Y, Laderas T, Maffit A, Kaempf A, Brady LK, et al. Reversible suppression of $T$ cell function in the bone marrow microenvironment of acute myeloid leukemia. Proc Natl Acad Sci U S A. 2020;117(25):14331-41.

3. Boddu P, Kantarjian H, Garcia-Manero G, Allison J, Sharma P, Daver N. The emerging role of immune checkpoint based approaches in AML and MDS. Leuk Lymphoma 2018;59(4):790-802.

4. Bewersdorf JP, Shallis RM, Zeidan AM. Immune checkpoint inhibition in myeloid malignancies: moving beyond the PD-1/PD-L1 and CTLA-4 pathways. Blood Rev 2020:100709.

5. Daver N, Garcia-Manero G, Basu S, Boddu PC, Alfayez M, Cortes JE, et al. Efficacy, safety, and biomarkers of response to azacitidine and nivolumab in relapsed/Refractory acute myeloid leukemia: a nonrandomized, Open-Label, Phase II Study. Cancer Discov 2019;9(3):370-83.

6. Beltra JC, Manne S, Abdel-Hakeem MS, Kurachi M, Giles JR, Chen Z, et al. Developmental Relationships of Four Exhausted CD8(+) T Cell subsets reveals underlying transcriptional and epigenetic landscape control mechanisms. Immunity 2020;52(5):825-41 e8.

7. Khan O, Giles JR, McDonald S, Manne S, Ngiow SF, Patel KP, et al. TOX transcriptionally and epigenetically programs CD8(+) $T$ cell exhaustion. Nature 2019;571(7764):211-8.

8. Pauken KE, Sammons MA, Odorizzi PM, Manne S, Godec J, Khan O, et al. Epigenetic stability of exhausted T cells limits durability of reinvigoration by PD-1 blockade. Science 2016;354(6316):1160-5.

9. Abdel-Hakeem MS, Manne S, Beltra JC, Stelekati E, Chen Z, Nzingha K, et al. Epigenetic scarring of exhausted T cells hinders memory differentiation upon eliminating chronic antigenic stimulation. Nat Immunol 2021;22(8):1008-19.

10. Milner JJ, Toma C, Quon S, Omilusik K, Scharping NE, Dey A, et al. Bromodomain protein BRD4 directs and sustains CD8 $\mathrm{T}$ cell differentiation during infection. J Exp Med 2021;218(8).

11. Kagoya Y, Nakatsugawa M, Yamashita Y, Ochi T, Guo T, Anczurowski M, et al. BET bromodomain inhibition enhances $T$ cell persistence and function in adoptive immunotherapy models. J Clin Invest 2016;126(9):3479-94.

Ethics Approval This study has been approved by the OHSU IACUC committee protocol IP00000907 "Immune-based therapeutic approaches for acute myeloid leukemia" Evan Lind PI.

http://dx.doi.org/10.1136/jitc-2021-SITC2021.734 